\author{
Ralahy Malinirina Fanjalalaina ${ }^{1,2 *}$, \\ Ravololoniaina Tiana ${ }^{2}$ and \\ Razafimahandry Henri Jean Claude ${ }^{1,2}$ \\ ${ }^{1}$ Department of Orthopaedy - Traumatology, Joseph \\ Ravoahangy Andrianavalona University Hospital of \\ Antananarivo, Madagascar \\ ${ }^{2}$ Faculty of Medicine, Antananarivo, Madagascar \\ Dates: Received: 05 October, 2015; Accepted: 08 \\ December, 2015; Published: 10 January, 2016 \\ *Corresponding author: Dr, Ralahy Malinirina \\ Fanjalalaina, Department of Orthopaedy - \\ Traumatology, Joseph Ravoahangy Andrianavalona \\ University Hospital of Antananarivo, Madagascar, Tel: \\ +261 (0) 3481039 98; E-mail: fafah.ralahy@gmail.com \\ www.peertechz.com
}

Keywords: Distal radius fractures; Intra-focal anesthesia; Kapandji; Pinning

\section{Research Article \\ Percutaneous Pinning of Distal Radius Fractures Using Hematoma Block: Outcomes in Our Center}

\section{Introduction}

Distal radius fractures account for $17 \%$ of all fractures. Menopausal women and men aged less than 40 are most concerned about. The treatment of distal radius fracture is a challenge in adult patients with the growing of extreme sports and the increasing of life expectancy. Non suitable treatment can also involve major functional loss in the wrist movements [1]. Surgical treatment avoids the secondary displacement of unstable fractures [1,2]. Kapandji-pinning is in this way widely practiced on account of its efficiency and relative reproducibility. In our center, hematoma block has been used for a long time in closed reduction at the emergency unit. Therefore, we found interesting to apply it while performing Kapandji-pinning the clinical outcomes of which are superior compared with nonoperative management. The purpose of this study was to demonstrate no difference in terms of patients' residual pain between Kapandjipinning and closed reduction associated with cast immobilization using both hematoma block in the treatment of unstable distal radius fractures. The secondary objective was to evaluate the surgical outcomes of Kapandji-pinning using intra-focal anesthesia.

\section{Methods}

This is a single-center prospective study from January to June 2013 enrolling patients managed for metaphyseal radius fracture with posterior displacement at the Emergency Unit. Selection criteria were patients having had extra-articular closed fractures with simple fracture line and posterior displacement as type 1 and 2 Kapandji fractures occurred after indirect injury. We included patients aged more than 15 without distinction as to sex. An informed consent was requested from patients then they were randomly assigned to Group 1 or Group 2 as regard to the drawn type of treatment. The first group underwent pinning of the fracture according to Kapandji technique. The second group was treated with closed reduction and cast immobilization. Treatment was performed by a single operator and hematoma block was used in both groups. Non-inclusion criteria were the presence of articular split, the allergy to local anesthesia products, open fractures and polytrauma. Patients lost to followup were excluded as well as those presented with any disabling disease. Radiography of the wrist (frontal and profile) was initially performed to assess the radial height. Eight milliliters of xylocaine $2 \%$ was injected at the site of fracture according to Biju S. and Aaron B technique [3]. In all procedures, the needle was laterally introduced then transversally slipped along the dorsal surface of the fracture till the top of the ulna. The needle was extracted afterwards, while slowly injecting the anesthetic agent (Figure 1A). The same procedure was realized on the ventral face of the fracture using the same entry (Figure 1B). Therefore, the periosteum was anesthetized. Finally, complementary $2 \mathrm{ml}$ radio guided anesthetic infiltration was performed at the future entrance of the pins. Fracture reduction was achieved under radioscopy 10 minutes after the anesthetic infiltration.

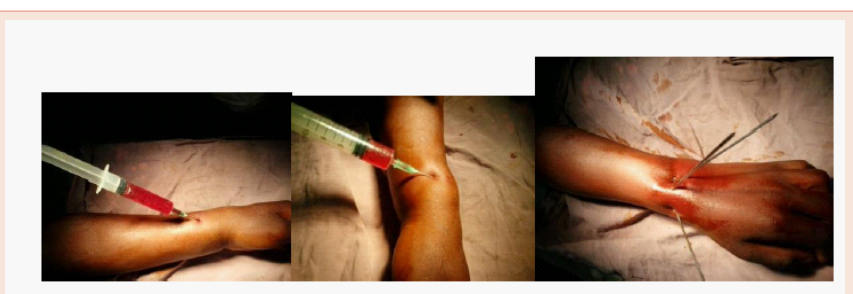

Figure 1: Anesthetic dorsal infiltration $(A)$ ad ventral infiltration $(B)$ of the hematoma block prior to Kapandji-pinning (C). 
Percutaneous intra-focal radio guided fixation of three pins was performed in Group 1 according to Kapandji technique (Figure 1C). Closed reduction and six weeks immobilization with long arm cast were realized in Group 2. X-ray images were taken after the one or another treatment. One gram of Paracetamol was given per os each 4 hours during the first 24 hours post-surgery associated with $50 \mathrm{mg}$ of Ketoprofene without contraindication each 12 hours. Patients were discharged the same day of surgery when no complication occurred. They were reviewed at day 7, 15, 30, 45, 60, 90. Passive movements were allowed the day after surgery for patients treated with Kapandjipinning as the pain can be tolerated. Functional rehabilitation had been started one month after treatment. The pins were removed on 6 weeks after surgery. In Group 2, functional rehabilitation had been started after the removal of the plaster cast. For all groups, operative and post-operative data were recorded. The outcomes were assessed on the presence of residual pain, the obtaining of anatomic reduction and stable fixation and the functional outcomes. Comparison between groups was done using Mann Whitney test and log rank test. The residual pain was evaluated using numeric pain rating scale ranging from 0 to 10 . A questionnaire was also given to evaluate the pain tolerance. The radial height was measured on post- operative X-ray. On day 7, 15 and 30, clinical and radiological complications were rated including edema, infection, algoneurodystrophy and secondary displacement. Clinical outcomes were appreciated over two month's follow-up using Herzberg functional score. The score ranged from 0 to 100 (excellent $=90$; good $=75$; medium $=60$; poor $<60$ ).

\section{Results}

Forty patients were enrolled on 18 in Group 1 (Kapandji treatment) versus 20 in Group 2 (no operative treatment) Two patients were lost to follow-up. Of the 38 included patients, 25 were female and 13 male. Mean age was 48 (17-77) years. Comparison between groups using Fisher test and Mann Whitney statistical test showed no difference for the distribution of age, sex and type of fracture (Table 1). No major incident was reported during the study. Overall mean radial height initially was 5,4mm (5,2 $\mathrm{mm}$ in Group 1 and 5,5mm in Group 2). Forty-four percent patients reported pain scored 2 (Figure 2) and no significant difference was observed between groups for pain scale [Mann-Whitney test: $\mathrm{U}=45,50$ - $\mathrm{p}$-value $=0,74 \mathrm{alpha}=0,05]$. In the pain tolerance questionnaire, none asked for another anesthetic agent. The most uncomfortable period was the fracture reduction time in all cases. Overall mean radial height of $12,7 \mathrm{~mm}$ was obtained after treatment $(14,4 \mathrm{~mm}$ in Group 1 and $11,6 \mathrm{~mm}$ in Group 2) (Figure 3). Over 60 days follow-up, 18 mild or moderate secondary displacements were registered of which 13 in Group 2. Two, four and five mild secondary displacement were observed respectively at day 30, 45, 60 in Group 1 and 11, 9, 8 in Group 2. No moderate displacement occurred in group 1 versus 4 and 5 at day 45 and day 60 respectively in Group 2. No severe displacement occurred in two groups. The survival curve on incidence of secondary displacement showed a difference between the two groups (Log-rank test: $\mathrm{X}^{2}=5,97$ - p-value $=0,01)($ Table 1$)$. Seventy percent overall good or excellent functional outcomes were obtained and no poor result was reported. In Group 2, 40\% had medium Herzberg score versus 22\% in Group 1 (Table 2). Clinical and radiological outcomes are summarized in Table 2.

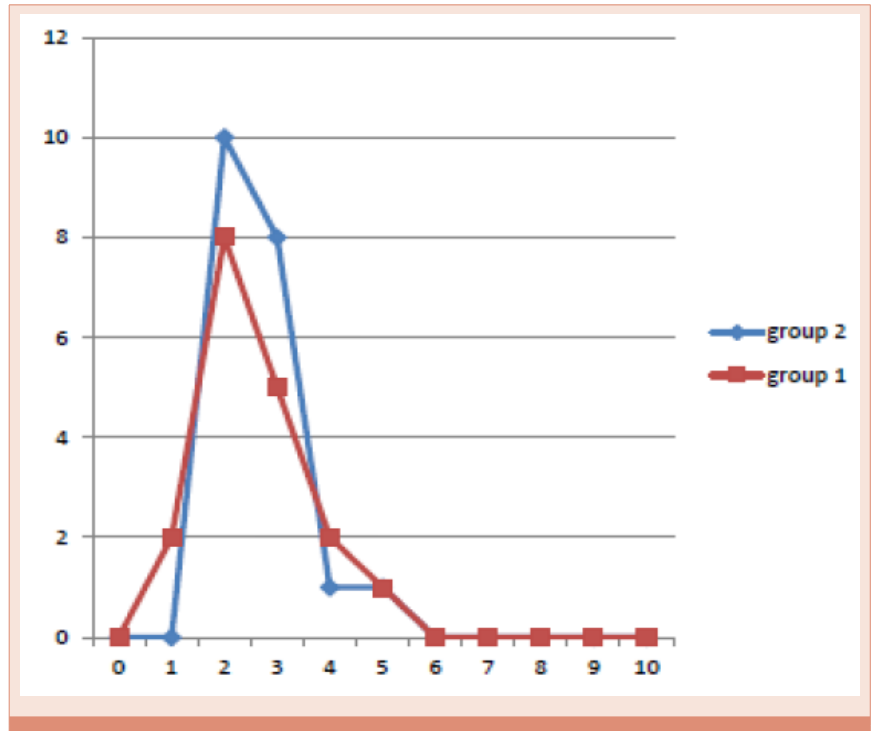

Figure 2: Value of residual pain in each group.

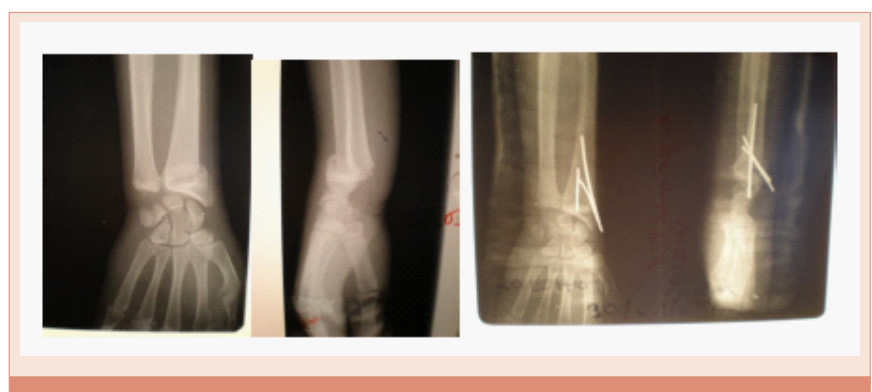

Figure 3: Pre-operative and post-operative X-ray images (A) of left extraarticular distal radius fracture in a 17 years-old patient treated with Kapandji -pinning $(B, C)$ under hematoma block.

Table 1: Epidemiological patients characteristics.

\begin{tabular}{|l|l|l|l|}
\hline & $\begin{array}{l}\text { Group 1 } \\
\mathbf{n = 1 8}\end{array}$ & $\begin{array}{l}\text { Group 2 } \\
\mathbf{n = 2 0}\end{array}$ & p-value \\
\hline Age & $\mathbf{4 7 [ 1 8 - 6 5 ]}$ & $\mathbf{4 8 [ 1 7 - 7 7 ]}$ & $\mathbf{0 , 4 5 5}$ \\
\hline $\begin{array}{l}\text { Gender } \\
\text { Male }\end{array}$ & 8 & 5 & \\
\hline $\begin{array}{l}\text { Female } \\
\text { Type of fracture } \\
\text { (Kapandji classification) } \\
\text { Type 1 } \\
\text { Type 2 }\end{array}$ & 10 & 15 & 0,30 \\
\hline Concomittant chronic disease & 7 & 5 & \\
\hline
\end{tabular}

\section{Discussion}

Intra-focal anesthesia is used by a lot of practitioners in Colles' fractures reduction $[3,4]$. In our series, the anesthetic agent used was Xylocaine 2\%. Almost half of the patients (44\%) had pain scored 2. The overall average pain scale was 2,86/10 (2-6); 2,55 in Kapandji group. Residual pain particularly occurred at the reduction time in both groups. Furia and et al. [5], in their study compared intra-focal anesthesia and sedation in closed reduction of distal radius fractures. The average pain scale was 2,7 that is close to our result. We therefore conclude that intra-focal anesthesia can be used in intra-focal pinning 
Table 2: Clinical and radiological outcomes.

\begin{tabular}{|c|c|c|c|}
\hline & $\begin{array}{l}\text { Group } 1 \\
n=18\end{array}$ & $\begin{array}{l}\text { Group } 2 \\
n=20\end{array}$ & p-value \\
\hline Mean residual pain score & 2,55 & 2,65 & 0,74 \\
\hline $\begin{array}{l}\text { Secondary displacement } \\
\text { mild } \\
\text { moderate } \\
\text { severe }\end{array}$ & $\begin{array}{l}5 \\
0 \\
0\end{array}$ & $\begin{array}{l}8 \\
5 \\
0\end{array}$ & 0,01 \\
\hline $\begin{array}{l}\text { Herzberg score } \\
\quad>90 \\
75-90 \\
60-75 \\
<60\end{array}$ & $\begin{array}{l}5 \\
9 \\
4 \\
0\end{array}$ & $\begin{array}{l}2 \\
10 \\
8 \\
0\end{array}$ & 0,12 \\
\hline
\end{tabular}

of distal radius fractures as closed reduction without involving more pain. Nevertheless, this type of anesthesia cannot be recommended in children as it requires the patient's active cooperation [6]. As the needle is set in direct contact with the site of fracture, practitioners mainly fear about infection risk while performing intra-focal anesthesia. But no infection occurred in our patients and actually no infection case reported in the literature. Moreover, Johnson and Noffsinger [7], in a comparative study between general anesthesia, intravenous regional anesthesia (Bier block) and intra-focal anesthesia asserted that intra-focal anesthesia when realized according to the usual asepsis rules does not increase the infection risk. As regard to the toxicity, intra-focal anesthesia is well-tolerated when the recommended dose is administrated. A team from the University of Colorado [8], had analyzed the variation curve of Lidocaine blood level after intra-focal administration of $2,4 \mathrm{mg} / \mathrm{kg}$ Lidocaine. First systemic peak appeared at fracture reduction time and maximal level of $1,100 \mathrm{ng} / \mathrm{ml}$ was observed 20 to $30 \mathrm{mn}$ after the infiltration. This level is far from the toxic level of $5,000 \mathrm{ng} / \mathrm{ml}$. Furthermore, the management proposed in our study namely Kapandji-pinning using intra-focal anesthesia lowers the cost of treatment and shortens the hospital stay. Hematoma block was only used in closed reduction and cast immobilization of distal radius fractures despite the high rate of secondary displacement. Kapandji pin fixation technique is easy to perform and offers good surgical outcomes (anatomic reduction and stable fixation leading to good functional results) [8]. Thus, it is the most operative technique used by orthopedists. Strohm and et al. [9], confirmed these good outcomes in a randomized study comparing intra-focal Kapandji-pinning and Willenegger- styloid pinning in the treatment of Colles' fractures. They used Martini score to define the best technique. Kapandji group resulted in excellent or good outcomes whereas Willenegger group resulted in only good or satisfactory results. In our series, $74 \%$ excellent or good Herzberg score were obtained in Kapandji group at 2 months. These results are also comparable to those reported in patients operated under general anesthesia. It must be noticed that functional results will be improved along the functional rehabilitation. Ruschel [10], effectively reported $72,1 \%$ excellent results at 3 months, $89,7 \%$ at 6 months and $96,6 \%$ at 12 months. The team used Gartland and Werley score to appreciate the functional results. Patients more than 55 years old or having impaction of the cortices more than $50 \%$ of the metaphyseal diameter should benefit from Kapandji-pinning treatment associated with cast immobilization [11], the non-adjunction of plaster cast in 5 patients treated with Kapandji technique may therefore explain the occurrence of mild secondary displacement in the Kapandji group. Carpal condyle thrust progressively leads to radial epiphysis impaction and secondary displacement [12], in our series, 18 secondary displacements were observed at 1 month of which $72 \%$ were treated with plaster cast.

\section{Conclusion}

Our study demonstrates the efficiency of hematoma block in the management of distal radius fractures with posterior displacement. Residual pain was tolerated both in non-operative and pinning treatment group. Hematoma block does not increase the infection risk if asepsis protocol is respected. The clinical outcomes after intrafocal anesthesia were similar to those reported after general anesthesia in the literature. Nevertheless, neither isolated closed reduction nor Kapandi-pinning can treat without any risk other types of fractures. Those need osteosynthesis with classical approach that is not feasible under intra-focal anesthesia. Only one technique cannot resolve all type of fractures at all.

\section{References}

1. Herzberg G, Dumontier C (2000) Symposium : les fractures fraîches du radius distal chez l'adulte. Rev Chir Orthop 86: 1585- 1588.

2. MacQuenn M, Caspers J (1988) Colle's fractures: does the anatomical result affect the final function. J Bone Joint Surg (Br) 70: 649- 651.

3. Sankar B, Aaron B (2005) Circumferential periosteal block in the reduction of distal radius fractures. Ann R Coll Surg Engl 87: 141.

4. Case RD (1985) Haematoma block a safe method of reducing Colles fractures. Injury 16: 469-470.

5. Furia JP, Alioto RJ, Marquardt JD (1997) The efficacy and safety of the hematoma block for fracture reduction in closed, isolated fractures. Orthopedics 20: 423-426.

6. Constantine E, Steele DW, Eberson C, Boutis K, Amanullah S, et al. (2007) The use of local anesthetic techniques for closed forearm fracture reduction in children: a survey of academic pediatric emergency departments. Pediatr Emerg Care 23: 209-211.

7. Johnson $P Q$, Noffsinger MA (1991) Hematoma block of distal forearm fractures. Is it safe? Orthop Rev 20: 977-979.

8. Meinig RP, Quick A, Lobmeyer L (1989) Plasma lidocaine levels following hematoma block for distal radius fractures. J Orthop Trauma 3: 187-191.

9. Castaing $J(1964)$ Recent fractures of the lower extremity of the radius in adults. Rev Chir Orthop Reparatrice Appar Mot 50: 581-696.

10. Strohm PC, Müller CA, Boll T, Pfister U (2004) Two procedures for kirschner wire osteosynthesis of distal radial fractures: A randomized trial Journal of Bone and Joint Surgery 86: 2621-2628.

11. Ruschel PH, Albertoni WM (2005) Treatment of unstable extra-articular distal radius fractures by modified intrafocal Kapandji method. Techniques in Hand and Upper Extremity Surgery 9: 7-16.

12. Trumble TE, Wagner W, Hanel DP, Vedder NB, Gilbert M (1998) M.Intrafocal (Kapandji) pinning of distal radius fractures with and without external fixation Journal of Hand Surgery 23: 381-394.

13. Guelmi K, Candelier G (1999) Comment nous traitons les fractures de l'extrémité inferieure du radius. Maîtrise Orthopédique $n^{\circ} 88$. 